\title{
Interactions of Alphavirus nsP3 Protein with Host Proteins
}

Tyler Lark, Forrest Keck and Aarthi Narayanan*

National Center for Biodefense and Infectious Diseases, School of Systems Biology, George Mason University, Fairfax, VA, United States

Alphaviruses are members of the Togaviridae family and are grouped into two categories: arthritogenic and encephalitic. Arthritogenic alphavirus infections, as the name implies, are associated with arthritic outcomes while encephalitic alphavirus infections can lead to encephalitic outcomes in the infected host. Of the non-structural proteins (nsPs) that the viruses code for, nsP3 is the least understood in terms of function. Alphavirus nsP3s are characterized by regions with significantly conserved domain structure along with regions of high variability. Interactions of nsP3 with several host proteins have been documented including, stress granule-related proteins, dead box proteins, heat shock proteins, and kinases. In some cases, in addition to the interaction, requirement of the interaction to support infection has been demonstrated. An understanding of the proteomic network of nsP3 and the mechanisms by which these interactions support the establishment of a productive infection would make alphavirus nsP3 an interesting target for design of effective medical countermeasures.

Keywords: alphavirus, nsP3, stress granules, DDX1/DDX3, IKK $\beta$, heat shock proteins, PI3K-Akt-mTOR, poly ADP

\section{INTRODUCTION}

Alphaviruses belong to the family Togaviridae and are divided into two categories: arthritogenic and encephalitic alphaviruses (Strauss and Strauss, 1994). The classification is indicative of their geographic locale and differences in pathogenesis and symptoms, namely New World alphaviruses tend to be associated with encephalitic phenotypes in the affected host while Old World alphaviruses are more likely to cause arthritic outcomes (Fros and Pijlman, 2016).

Alphaviruses are spherical in shape, $\sim 65 \mathrm{~nm}$ in diameter, and enveloped with an icosahedral capsid (Jose et al., 2009). The genome consists of an $\sim 11.5-\mathrm{kb}$ positive sense, single-stranded RNA with a $5^{\prime}$ cap and 3' poly(A) tail (Garmashova et al., 2007), which encodes five structural (capsid, E1-E3, 6K) and four non-structural proteins (nsPs: nsP1-4). With regard to functionality associated with structural proteins, capsid binds to viral RNA and stimulates nucleocapsid assembly and RNA synthesis; E1 stimulates the fusion of the viral membrane with the endosomal membrane; E2 aids in the binding to the target receptor and viral uptake via receptor-mediated endocytosis (Dubuisson and Rice, 1993); E3 aids in the folding of the precursor E2 protein and in the activation of E1 (Lobigs et al., 1990); 6K forms ion channels within the host cell and aids in trafficking, assembly (Lusa et al., 1991), and budding of the virus. As for the nsPs, nsP1 is a membraneanchored protein that is responsible for the synthesis and capping of RNA (Wang et al., 1991); nsP2 acts as a RNA helicase (Gomez de Cedrón et al., 1999) and cleaves the non-structural polyprotein into its individual elements (Strauss et al., 1992); nsP4 acts as an RNA polymerase (Hahn et al., 1989). Little is known about the function of nsP3 other than an appreciation of its requirement for 
RNA synthesis (Strauss and Strauss, 1994; Garmashova et al., 2007; Fros and Pijlman, 2016). This review will elucidate the interactions of nsP3 with host proteins in order to better understand its role in the life cycle of alphaviruses. Where possible, differences in protein interactions between arthritogenic and encephalitic alphaviruses will be highlighted.

\section{nsP3 STRUCTURE}

The nsP3 is an $\sim 60 \mathrm{kDa}$-sized protein that can be divided into three different domains: macrodomain, alphavirus unique domain, and hypervariable region (Figure 1; Rupp et al., 2015). The macrodomain is located on the N-terminus of the protein and is a highly conserved sequence of $\sim 150$ amino acids, which is also found in other viruses such as coronaviruses (Beitzel et al., 2010; Rungrotmongkol et al., 2010). The structure of the this domain consists of a six-stranded, twisting, centrally located $\beta$-sheet that is surrounded by four $\alpha$-helices, three on one side and one on the other. This $\beta$-sheet, $\alpha$-helix arrangement is preserved among both arthritogenic and encephalitic alphaviruses. Within this region, there is a crevice for the interaction with adenosine diphosphate ribose (ADP ribose) (Karras et al., 2005; Malet et al., 2009; Park and Griffin, 2009a; Foy et al., 2013), whose role will be expounded upon in a later section. The alphavirus unique domain comprises the middle third of nsP3 and is maintained only within alphaviruses (Rupp et al., 2015). This segment of the protein contains many serine and threonine residues, forming two parallel $\beta$-sheets and antiparallel $\alpha$-helices. This domain also includes a zinc-binding domain which is important in cleaving of the P2/3 polyprotein, infectivity, and RNA production (Shin et al., 2012; Rupp et al., 2015). The hypervariable region is located on the C-terminus of the protein. Unlike the macrodomain and alphavirus unique domain, the hypervariable domain is not conserved among alphaviruses in sequence or structure. This segment is rich in serine and threonine residues and offers numerous sites for phosphorylation (Foy et al., 2013). The length of this region can vary based on the alphavirus, and even those which have a similar length, such as Venezuelan Equine Encephalitis Virus (VEEV) and Sindbis Virus (SINV), differ in the sequence of amino acids that make up this section. This variability could account for, or at least play a role, in the difference in pathogenicity and protein interactions of nsP3 in the context of different viruses (Varjak et al., 2010). However, it has been noted that there is a certain level of conservation among several alphaviruses in the C-terminal domain. Removal of this conserved sequence area in Semliki Forest Virus (SFV) showed a decrease in infectivity of the virus, attesting to its importance in the infectious process (Vihinen et al., 2001).

\section{nsP3 INTERACTIONS}

\section{ADP-Ribose-Binding Site}

The ADP-ribose-binding site of alphaviruses is conserved in structure, but amino acid residues may differ between arthritogenic and encephalitic viruses. The ability to bind

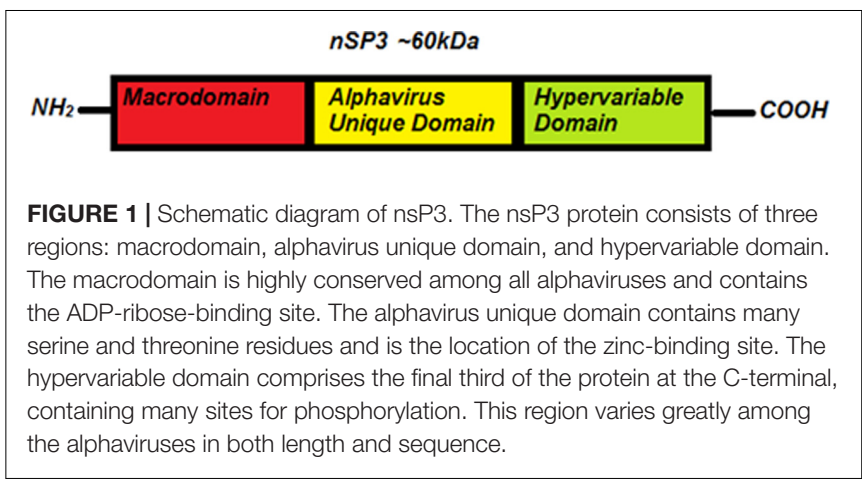

ADP ribose, as well as RNA, was shown to be comparable between VEEV and Chikungunya Viruses (CHIKV) (Malet et al., 2009), although VEEV has stronger affinity than CHIKV (Park and Griffin, 2009b). Activity as an ADP-ribose 1"-phosphate phosphatase activity was similar between the two viruses. However, SFV phosphatase activity was detected at a much lower level (Malet et al., 2009), suggesting that differences in activity may vary from species to species rather than being able to group by phosphatase activity solely on arthritogenic and encephalitic classification. There is also no correlation in ability to bind monomeric ADP ribose and poly ADP ribose. Studies with SFV and Hepatitis E virus, another macrodomain containing virus, show that both effectively bind poly ADP ribose, but Hepatitis $\mathrm{E}$ virus has an affinity for monomeric ADP ribose while SFV has no attraction (Neuvonen and Ahola, 2009). The role of binding to poly or monomeric ADP ribose in the life cycle of the viruses is unclear. For CHIKV, the ability to hydrolyze monomeric ADP ribose was shown to be essential for both viral replication and virulence. Mutant nsP3 viruses that lacked the ability to either bind or hydrolyze ADP ribose had a significant decrease in viral titers in in situ experiments. In vivo studies revealed that hindrance of both binding and hydrolyzing capabilities greatly reduced virulence in mice, but interestingly, hydrolyzing mutants maintained virulence (McPherson et al., 2017). In eukaryotic cells, monomeric ADP ribosylation influences processes such as immune response and metabolism, and poly ADP ribosylation is an important post-translational modification event. Poly ADPribose polymerases (PARP) facilitate this event. There are a wide number of PARPs and they respond to different stimuli within the cell. PARP-1 is stimulated by damage to cellular DNA and is a key factor in the survival of the cell (Schreiber et al., 2006). When mutations were made in the ADP-ribose-binding site of nsP3 of SINV, there was no effect on the virus' ability to bind poly ADP ribose in mouse neuronal cells. However, the virus' ability to replicate was hindered. The ability of the virus to form replication complexes and synthesize RNA was greatly diminished. An increase in neuronal cell death was higher in these mutated nsP3 viruses as compared to the wild type (Malet et al., 2009). Further investigation into this showed that PARP-1 is closely associated with nsP3 functionality. Co-immunoprecipitation of PARP-1 in infected cells revealed that the polymerase was associated with $\mathrm{nsP} 3$ in the replication complex. The other nsPs are not required for association of PARP-1 with nsP3, and enzymatic activity 
did not affect viral replication. Surprisingly, PARP-1 was not associating with the ADP-ribose-binding site, but rather with the C-terminal domain. It is thought that this recruitment of PARP1 is important in stabilizing the replication complex or for the recruitment of other host proteins (Park and Griffin, 2009a).

\section{Stress Granule-Related Proteins}

During cellular stress, the translation of mRNA can be interrupted, leading to their cytoplasmic accumulation. This cytoplasmic accumulation of mRNPs and stress proteins results in the formation of stress granules. The function of these complexes is either to carry out the unfinished translation of the mRNA or to degrade the molecule (Buchan and Parker, 2009). Alphavirus nsP3 has been documented as interacting with proteins related to stress granule formation. There are differences in how arthritogenic and encephalitic alphavirus nsP3 proteins interact with components of the stress granules. Arthritogenic alphavirus nsP3 interacts with the host protein Ras-GTPase activating SH3-binding domain protein (G3BP). G3BPs are RNAbinding proteins that aid in the organization of the stress granule. Arthritogenic nsP3 interacts with G3BPs through a region on the C-terminal domain containing two FGDF motifs, two phenylalanine residues which are separated by a glycine and aspartate residue. This area was found to be conserved in multiple arthritogenic viruses, suggesting that they use the same mechanism to recruit G3BPs. Most other FGDF-containing proteins contain only a single motif as compared to the two found in the alphavirus. Having two may ensure the rapid sequestering of G3BP before stress granules can be formed (Panas et al., 2014, 2015; Schulte et al., 2016). The interaction of nsP3 and G3BP has been extensively studied in SINV, CHIKV, and SFV. G3BP1 and G3BP2 are found spread throughout the cell, and soon after infection those are localized to nsP3 containing replication complexes in SINV infections (Frolova et al., 2006; Gorchakov et al., 2008). Studies with CHIKV show that this attraction leads to the formation of stress granule-like complexes that differ in composition to true stress granules and are non-functional. During later parts of replication, G3BP is shifted away from the replication complex associated with the cell's membrane toward the cytoplasm. Interestingly, it was found that reducing the level of G3BP1 and G3BP2 resulted in a decrease in the amount of viral RNA. There was a detectable level of the two proteins associated with the viral replication complex at the cell membrane and was noted to be involved in the switch from translation to minusstrand RNA synthesis (Cristea et al., 2006; Fros et al., 2012; Scholte et al., 2015; Fros and Pijlman, 2016; Kim et al., 2016). This finding is contradictory to another study with SINV that found depleting G3BP1 and G3BP2 levels led to an increase in viral RNA production (Cristea et al., 2010). In SFV infections, the G3BP proteins were also associated with the replication complex early on in infection (Panas et al., 2012). Mosquitoes are natural vectors for the transmission of alphaviruses. Mosquito cells and live mosquitoes, Aedes albopictus, were infected with CHIKV to determine whether a similar association with stress granules existed. The mosquito G3BP homolog, Rasputin, was found to be localized to nsP3 foci. Silencing of Rasputin in in vivo oral infections lead to a reduction in viral production, whereas the same silencing in in vitro studies did not affect viral replication. It has been proposed that interaction between nsP3 and Rasputin may interfere with immune responses (Fros et al., 2015).

Whereas arthritogenic alphavirus nsP3 has been shown to interact with G3BP proteins, encephalitic alphavirus nsP3 did not co-localize with the protein. VEEV forms nsP3-associated complexes, but the hypervariable domain was shown to not bind to G3BP. Replacing the hypervariable domain of SINV with that of VEEV disrupted the virus' ability to associate with G3BP (Foy et al., 2013). Rather, it was shown that VEEV associates with Fragile-X-related (FXR) proteins. FXR exists in two forms, FXR1 and FXR2, which are homologs of Fragile-X mental retardation protein. These proteins formed heterodimers and associated with mRNPs in the formation of stress granules (Tamanini et al., 1999, 2000; Anderson and Kedersha, 2008). Inhibition of both FXR proteins reduced the level of virus being produced, and inhibition of FMRP exacerbated the effect. The binding of nsP3 to the Fragile-X proteins promoted the formation of the viral replication complex and RNA replication. There was also an accumulation of viral G RNA in these complexes, which was needed for translation of the early polyproteins (Kim et al., 2016).

Interestingly, Eastern Equine Encephalitis Virus (EEEV) has recently been shown to be capable of utilizing both FXR and G3BP proteins. The HVD of EEEV contains a single-binding site for both sets of proteins and the binding of one is independent of the other. As a result of this independent binding, inhibition of only one of the proteins did not result in a decrease in viral replication and dual inhibition was critical for inhibition. It was suggested that binding to both FXR and G3BP proteins played a role in the high pathogenicity of EEEV and may show the evolutionary branching of the HVD from the G3BP binding of the arthritogenic alphaviruses and the FXR binding of encephalitic alphaviruses (Frolov et al., 2017).

\section{Amphiphysins}

Amphiphysins are a family of proteins that are involved in various cellular processes such as endocytosis and membrane trafficking. Amphiphysin 1 and 2 are predominately found in neuronal cells and are crucial for the endocytosis of clathrinmediated vesicles in synapses. They facilitate this process through an interaction with dynamin via their SH3 domain (Wigge and McMahon, 1998). The SH3 domain is a non-catalytic domain that is found on numerous signaling proteins (Weng et al., 1995). Alphaviruses are able to interact with this $\mathrm{SH} 3$ domain to recruit amphiphysin to the site of replication complexes (Neuvonen et al., 2011). While nsP3 interacts with the same SH3-binding site as dynamin, it does so with a higher affinity (Tossavainen et al., 2016). Infections with SFV and SINV showed that during early time points amphiphysin 1 localized to the replication complex and to the plasma membrane in later time points. Amphiphysin 2 localized in a similar pattern at early time points, but in later time points, it was observed in cytopathic vacuoles. This difference in localization at the different time points could be attributed to the differences in functionalities of the two proteins. Inhibiting this localization through mutations in the $\mathrm{SH} 3$ domain was shown to inhibit the production of viral RNA (Neuvonen et al., 2011). Their importance in viral replication could be 
linked to the association of the alphavirus replication complex to membranes. The replication complex is initially formed on the plasma membrane after entry and is transported to early endosomes and lastly ends up in invaginations of the lysosomal membrane. nsP3 is located on the surface of these spherules in order to interact with other proteins (Froshauer et al., 1988; Peränen and Kääriäinen, 1991; Jürchott et al., 2003; Spuul et al., 2011). The involvement of amphiphysins in endocytosis and membrane trafficking may suggest that they are important in the formation of this invagination of lysosomal membranes to house the viral replication complex.

\section{Y-Box-Binding Protein 1 (YBX1)}

Y-box-binding protein 1 (YBX1) was shown to be associated with nsP3 of SINV. YBX1 was coupled to nsP3 in complexes located on endosomal membranes as well as in the nucleus (Gorchakov et al., 2008). However, the importance of this interaction has not been elucidated. YBX1 is capable of shuttling between the cytoplasm and the nucleus and has been implicated in numerous cellular functions. In the nucleus, it controls the transitioning of the cell cycle from G1 to S phase (Jürchott et al., 2003), DNA replication and repair, and transcriptional activation. In the cytoplasm, YBX1 aids in chaperoning and translation of mRNA and is a component of mRNPs (Matsumoto and Wolffe, 1998; Lu et al., 2005; Eliseeva et al., 2011). YBX1 is organized as three domains: a variable $\mathrm{N}$-terminal domain, cold shock domain, and C-terminal domain. The cold shock and C-terminal domain are capable of binding ssRNA, ssDNA, DNA, and proteins (Kloks et al., 2002; Mihailovich et al., 2010). Looking at YBX1 interactions with other viruses does not clear the picture as to its role in binding to alphavirus nsP3. In Dengue virus infections, YBX1 inhibits the production of viral particles. In this association, YBX1 binds to the $3^{\prime}$ untranslated region and suppresses translation of the viral RNA (Paranjape and Harris, 2007). It remains unclear whether the lack of a poly(A) tail in Dengue virus could explain differential utility of YBX1 in this case as compared to alphaviruses. However, when looking at Hepatitis $\mathrm{C}$ virus, YBX1 acts as both a stimulant and a repressor. The association of YBX1 with NS3/4a is necessary for an efficient replication of viral RNA. There is an inverse correlation between the number of viral particles produced and the amount of YBX1 present. This shows that YBX1 is an important regulator in controlling Hepatitis $\mathrm{C}$ replication and virus assembly (ChatelChaix et al., 2011). The human polyomavirus JC virus makes use of YBX1 as a transcriptional activator to facilitate its survival (Raj et al., 1996). YBX1 has been shown to aid in cell survival during stress (Lu et al., 2005) and inhibits MHCII molecules (Didier et al., 1988; Ting et al., 1994). With a prominent nuclear phase involved in polyomavirus life cycle, the role of YBX1 can have different implications as an enabler of viral transcription as contrasted with alphaviruses where the life cycle is predominantly cytoplasmic. Taking these into account, it is possible that YBX1 may play a role in the replication of alphaviruses or be involved in keeping the cell alive by suppressing immune responses. More studies need to be done to determine the functional significance of the YBX1:nsP3 interaction in alphavirus life cycle.

\section{Heat Shock Proteins}

Heat shock proteins are regulatory proteins that play essential roles during cellular stress. They act as chaperones and maintain proper folding of proteins that would otherwise denature during stress responses. There are multiple members of the heat shock protein family whose function can be both tissue and organelle specific (Jee, 2016). The Hsp70 and Hsp90 family have been show to play a role in the life cycle of alphaviruses through nsP3 interactions. The Hsp70 family consists mainly of two proteins: Hsc70 and Hsp70. Hsc70 is a constitutively expressed heat shock cognate protein. It functions in aiding in the uncoating of clathrin-coated vesicles, transporting proteins to various organelles, and targeting proteins to lysosomes. Hsp70, unlike Hsc70, is only expressed during stress conditions. The interactions that Hsp70 has are very similar to Hsc70, with only a few differences, such as associating with immunogenic peptides (Goldfarb et al., 2006; Liu et al., 2012). A study looking at host protein associations with SINV nsP3 showed an interaction with Hsc70 (Gorchakov et al., 2008). The functional relevance of the interaction has yet to be determined, but looking at other viruses shows that Hsc70 is important for the life cycle of viruses. Rotaviruses interact with Hsc70 at the cell surface after binding to their receptor. This interaction mediates the entry of the virus into the cytoplasm of the cell (Zárate et al., 2003). The Hepatitis $B$ virus reverse transcriptase is normally activated by Hsp90, but studies have shown that Hsc70 is also able to act as an enzymatic catalyst to trigger the reverse transcriptase (Beck and Nassal, 2003). In HIV, Hsc70 and Hsp70 aid in the transport of viral polyproteins to the plasma membrane (Gurer et al., 2002). Tombusviruses utilize Hsp70 to insert the viral replicase into the intracellular membranes and efficiently replicate (Serva and Nagy, 2006; Wang et al., 2009). An interaction with the SINV $\mathrm{nsP} 3$ for viral entry seems unlikely, as the protein is not expressed on the surface of the virion, but a role in viral replication could be plausible.

Hsp90 aids in the folding and activation of numerous signaling proteins involved in cellular proliferation and survival, such as src family kinases and Raf proteins (Verma et al., 2016). Hsp90 has been shown to interact with the nsP3 of CHIKV to promote viral replication. Hsp $90 \beta$ was co-immunoprecipitated with nsP3 during infections, another Hsp90 was shown to interact with nsp4. Inhibition of Hsp90 was shown to decrease viral RNA and protein production (Rathore et al., 2014). A follow-up study revealed that Hsp90 aids in replication by stabilizing the nsP2 protein (Das et al., 2014). Hsp90 is essential for replication in other viruses as well, aiding in viral RNA polymerase synthesis in flock house virus (Castorena et al., 2007) and stabilizes the $\mathrm{L}$ protein of the viral polymerase for vesicular stomatitis virus (Connor et al., 2007). Whether similar functionalities are associated with the alphavirus nsP3 interaction remain to be determined.

\section{PI3K-Akt-mTOR}

The PI3K-Akt-mTOR pathway plays a crucial role in various cellular processes such as cell growth and survival. Phosphatidylinositol-3 kinases (PI3K) is a family of kinases that 
phosphorylate phosphoinositides to generate phoshatidylinositol-3,4,5-trisphosphate (PIP3). PI3Ks are activated by the binding of a tyrosine kinase receptor (Owonikoko and Khuri, 2013). PIP3 binds to and activates Akt, which is also phosphorylated by PDK1 (Vara et al., 2004). Akt is then able to activate mammalian target of rapamycin (mTOR), which is a regulator of protein synthesis and cell survival (Memmott and Dennis, 2009). The PI3K-Akt-mTOR pathway has been shown to be exploited by numerous viruses, normally to prevent an anti-apoptotic response (Dunn and Connor, 2012; Diehl and Schaal, 2013). nsP3 of SFV was shown to activate Akt directly, but only when associated with the plasma membrane. The hyperphosphorylated region of the HVD region of nsP3 was indicated as being critical for the interaction (Thaa et al., 2015). Activation of this complex was shown to be necessary for the internalization of the replication complex that assembled at the plasma membrane (Spuul et al., 2010). Wild type nsP3 of SFV was able to directly activate Akt, while activation of Akt in SFV $\triangle 50$, a mutant strain with a deletion of residues 319-368 in nsP3 hyperphosphorylated region, was suppressed. Interestingly, CHIKV infection only moderately activated Akt and was dependent upon PI3K activation of Akt. The activation of Akt by PI3K did not stimulate the internalization of replication complexes for CHIKV (Thaa et al., 2015). In arthropods, SINV infection led to the activation of the PI3K-Akt-TOR pathway which contributed to an uptick in translation through TOR activation of 4E-BP1, which activates eIF4E, a protein necessary for cap-dependent translation. Translation of both viral and host mRNA is promoted through this activation. Activation of $4 \mathrm{E}-\mathrm{BP} 1$ is not evident in vertebrates, as alphaviruses can inhibit host translation during infection (Patel and Hardy, 2012). In in vitro studies using human cells, SINV was shown to suppress PI3K-Akt-mTOR late in infection. Viral replication was not inhibited by using inhibitors of PI3K and mTOR (Mohankumar et al., 2011). Taken together, these data suggest that the PI3KAkt-mTOR pathway may not be necessary for the production of virions, but is required for the internalization of the replication complex.

\section{DDX1/DDX3}

DEAD box proteins are a family of ATP-dependent RNA helicases that function in multiple steps of RNA metabolism. Along with the involvement in cellular processes with RNA, DEAD box proteins have also been shown to play a role in antiviral immune response. DDX1 is involved in the sensing of dsRNA and production of an interferon response. Also, it binds to the p65 subunit of NFKB to enhance inflammatory cytokine production (Fullam and Schröder, 2013). DDX3 also has antiviral properties. DDX3 can recognize viral RNA and generate a response through the RIG-I-like receptor family to generate type I IFN. DDX3 binds to IKKE, which phosphorylates DDX3 and recruits IRF3 (Valiente-Echeverría et al., 2015). Phosphorylation of DDX3 by TBK1 promotes the recruitment of DDX3 to the interferon $\beta$ promoter and induction of interferon production. DDX3 has also been shown to sense viral dsDNA and promote generation of interferon (Ariumi, 2014). Using a mass spectrometry approach, VEEV nsP3 was shown to associate with both DDX1 and DDX3. A knockdown of both helicases resulted in a drop in viral replication. It has been suggested that DDX1 and DDX3 may play a role in the unwinding of viral RNA for replication and/or translation in stress granules (Amaya et al., 2016). DDX1 and DDX3 have been shown to be exploited by numerous other viruses. DDX1 promotes replication in Coronaviruses (Xu et al., 2010) and transcription in JC virus (Sunden et al., 2007). In HIV-1, DDX3 aids in the shuttling of Rev between the nucleus and the cytoplasm and DDX1 acts as a co-factor for the function of Rev (Li et al., 2005). DDX3 is used to stimulate the replication of Hepatitis $\mathrm{C}$ virus (Oshiumi et al., 2010; Wang and Ryu, 2010) and is sequestered in Hepatitis $\mathrm{B}$ infections to prevent an immune response (Ariumi et al., 2007). An interesting functionality associated with DDX3 as a shuttling protein in the context of alphavirus infectious cycle will be to evaluate whether it has any role to play in the nuclear localization of the capsid protein, and hence influence control of transcription.

\section{IKK $\beta$}

$\mathrm{NF} \kappa \mathrm{B}$ is a transcription factor that is involved in the activation of genes participating in immune and inflammatory responses, cell proliferation, and survival (Israël, 2010). NFкB is activated in two different manners: a canonical and alternative pathway. Focusing on the canonical pathway, activation begins with the binding of proinflammatory, IL-1 and TNF $\alpha$, to a Toll-like receptor (Lawrence, 2009). This leads to the recruitment of adaptor proteins, such as TNF receptor associated factors, to phosphorylate the IKK complex. The IKK complex consists of the three subunits IKK $\alpha$, IKK $\beta$, and IKK $\gamma$ (Amaya et al., 2014). IKK $\beta$ phosphorylates the inhibitory $\kappa B$ proteins associated with the p65/p50 subunits of $\mathrm{NF \kappa B}$. I $\mathrm{B} \alpha$ is then tagged for ubiquitination, releasing the two NFKB subunits for nuclear transportation (Lawrence, 2009). The NFKB pathway is an oftenutilized pathway for viruses, such as HTLV-1 (Hai et al., 2006; Hiscott et al., 2006) and Hepatitis B virus (Santoro et al., 2003). A recent study has shown that VEEV nsP3 associated with the IKK $\beta$ subunit of the IKK complex, potentially through the HVD region to activate the canonical pathway of NFKB. Viral replication triggered the activation of TLR3, activating the IKK $\beta$ complex. Inhibition of IKK $\beta$ function led to a broad spectrum decrease in the replication of encephalitic alphavirus in cell culture models and VEEV, in vivo (Amaya et al., 2014).

\section{Applications}

Alphavirus nsP3 interactions with host proteins have been demonstrated to play an integral part in the viral replication process. In several of these documented interactions, while descriptive features of the interactions may be available, mechanistic details of functional requirements of such interactions remain to be elucidated. While inferences can be made by looking at other viruses that utilize the same proteins and initial hypotheses of possible functions as related to the establishment of a productive infection can be proposed, it is important to understand that many of these viruses differ greatly from alphaviruses in genomic makeup and life cycle, raising the possibility that the purpose and mechanism of 
those interactions may be different. As elaborated in several instances above, the same host protein may play opposing roles in the context of different viruses, thus underscoring the very important need to understand how such host proteins influence an infectious cycle within the context of viral biology. Many such contrasting functions may also be manifestations of not just the simple interaction between the target viral and host proteins, but involve larger dynamic protein networks. These multiple interactions of nsP3 and critical importance of the host protein interaction networks offer the additional potential to diversify the target portfolio for development of therapeutic candidates and identification of candidates that are supportive of broad spectrum inhibition. Of relevance, the inhibition of many of these interactions has been shown to impede viral replication. Use of RK-33, a small molecule inhibitor of DDX3, decreased infectious viral titers for VEEV (Amaya et al., 2016). The use of an IKK $\beta$ inhibitor had a negative effect on the ability of VEEV to efficiently replicate (Amaya et al., 2014). Cell lines with a knockout of FXR inhibited VEEV replication, with a similar effect noticed for SINV and CHIKV in cells with knockouts of G3BP1 and G3BP2 (Kim et al., 2016). nsP3 is an important contributor to the virulence of alphaviruses. Multiple studies have shown that creating mutations or deletions within the protein hinders the virus's ability to effectively replicate in the host. In SFV, deletions in the C-terminal region hampered the production of subgenomic RNA and establishment of infection, results (Varjak et al., 2010) which were also a product of experiments with mutations and deletions in the hypervariable region (Galbraith et al., 2006). Mutations in the hypervariable region also resulted in a virulence in mice inoculated both intranasally and intramuscularly. Mutations inserted into the nsP3 of SINV show a similar result, reduction in viral RNA being produced (LaStarza et al., 1994; Dé et al., 2003). The use

\section{REFERENCES}

Amaya, M., Brooks-Faulconer, T., Lark, T., Keck, F., Bailey, C., Raman, V., et al. (2016). Venezuelan equine encephalitis virus non-structural protein 3 (nsP3) interacts with RNA helicases DDX1 and DDX3 in infected cells. Antiviral Res. 131, 49-60. doi: 10.1016/j.antiviral.2016.04.008

Amaya, M., Voss, K., Sampey, G., Senina, S., de la Fuente, C., Mueller, C., et al. (2014). The role of IKK $\beta$ in Venezuelan equine encephalitis virus infection. PLOS ONE 9:e86745. doi: 10.1371/journal.pone.0086745

Anderson, P., and Kedersha, N. (2008). Stress granules: the Tao of RNA triage. Trends Biochem. Sci. 33, 141-150. doi: 10.1016/j.tibs.2007. 12.003

Ariumi, Y. (2014). Multiple functions of DDX3 RNA helicase in gene regulation, tumorigenesis, and viral infection. Front. Genet. 5:423. doi: 10.3389/fgene.2014. 00423

Ariumi, Y., Kuroki, M., Abe, K., Dansako, H., Ikeda, M., Wakita, T., et al. (2007). DDX3 DEAD-box RNA helicase is required for hepatitis C virus RNA replication. J. Virol. 81, 13922-13926. doi: 10.1128/JVI.01517-07

Beck, J., and Nassal, M. (2003). Efficient Hsp90-independent in vitro activation by $\mathrm{Hsc70}$ and Hsp40 of duck hepatitis B virus reverse transcriptase, an assumed Hsp90 client protein. J. Biol. Chem. 278, 36128-36138. doi: 10.1074/ jbc.M301069200

Beitzel, B. F., Bakken, R. R., Smith, J. M., and Schmaljohn, C. S. (2010). Highresolution functional mapping of the Venezuelan equine encephalitis virus genome by insertional mutagenesis and massively parallel sequencing. PLOS Pathog. 6:e1001146. doi: 10.1371/journal.ppat.1001146 of massive parallel sequencing in VEEV has allowed for the identification of regions within the nsP3 that are tolerant of mutations, the C-terminal region, and those that are intolerant of mutations, the N-terminal region (Beitzel et al., 2010). Given the significance that $\mathrm{nsP} 3$ has in fostering a productive viral infection, more studies need to be performed to determine the exact mechanisms of interactions nsP3 has with host proteins, elucidate protein interaction networks as variables of stages of viral infectious cycle, and understand their relevance in the establishment of a productive infection.

\section{AUTHOR CONTRIBUTIONS}

TL, FK, and AN wrote the article. TL was responsible for collation of information regarding nsP3 interaction partners. FK assisted with article preparation and formatting, and in addition to contributing to the written body. AN worked with TL and FK to compose the article and final review.

\section{FUNDING}

$\mathrm{AN}$ is the principal investigator on a NIH R15 grant that was used to fund this work (R15AI123993).

\section{ACKNOWLEDGMENTS}

The authors would like to thank members of the Narayanan Laboratory for critical review of the manuscript. They would also like to acknowledge the funding support afforded by a NIH R15 grant to AN.

Buchan, J. R., and Parker, R. (2009). Eukaryotic stress granules: the ins and out of translation. Mol. Cell 36:932. doi: 10.1016/j.molcel.2009.11.020

Castorena, K. M., Weeks, S. A., Stapleford, K. A., Cadwallader, A. M., and Miller, D. J. (2007). A functional heat shock protein 90 chaperone is essential for efficient flock house virus RNA polymerase synthesis in Drosophila cells. J. Virol. 81, 8412-8420. doi: 10.1128/JVI.00189-07

Chatel-Chaix, L., Melançon, P., Racine, M.-E., Baril, M., and Lamarre, D. (2011). Y-box-binding protein 1 interacts with hepatitis $C$ virus NS3/4A and influences the equilibrium between viral RNA replication and infectious particle production. J. Virol. 85, 11022-11037. doi: 10.1128/JVI.00719-11

Connor, J. H., McKenzie, M. O., Parks, G. D., and Lyles, D. S. (2007). Antiviral activity and RNA polymerase degradation following Hsp90 inhibition in a range of negative strand viruses. Virology 362, 109-119. doi: 10.1016/j.virol.2006.12. 026

Cristea, I. M., Carroll, J. W. N., Rout, M. P., Rice, C. M., Chait, B. T., and MacDonald, M. R. (2006). Tracking and elucidating alphavirus-host protein interactions. J. Biol. Chem. 281, 30269-30278. doi: 10.1074/jbc.M603 980200

Cristea, I. M., Rozjabek, H., Molloy, K. R., Karki, S., White, L. L., Rice, C. M., et al. (2010). Host factors associated with the Sindbis virus RNA-dependent RNA polymerase: role for G3BP1 and G3BP2 in virus replication. J. Virol. 84, 6720-6732. doi: 10.1128/JVI.01983-09

Das, I., Basantray, I., Mamidi, P., Nayak, T. K., Pratheek, B. M., Chattopadhyay, S., et al. (2014). Heat shock protein 90 positively regulates Chikungunya virus replication by stabilizing viral non-structural protein nsP2 during infection. PLOS ONE 9:e100531. doi: 10.1371/journal.pone.0100531 
Dé, I., Fata-Hartley, C., Sawicki, S. G., and Sawicki, D. L. (2003). Functional analysis of nsP3 phosphoprotein mutants of Sindbis virus. J. Virol. 77, 13106-13116. doi: 10.1128/JVI.77.24.13106-13116.2003

Didier, D. K., Schiffenbauer, J., Woulfe, S. L., Zacheis, M., and Schwartz, B. D. (1988). Characterization of the cDNA encoding a protein binding to the major histocompatibility complex class II Y box. Proc. Natl. Acad. Sci. U.S.A. 85, 7322-7326. doi: 10.1073/pnas.85.19.7322

Diehl, N., and Schaal, H. (2013). Make yourself at home: viral hijacking of the PI3K/Akt signaling pathway. Viruses 5, 3192-3212. doi: 10.3390/v5123192

Dubuisson, J., and Rice, C. M. (1993). Sindbis virus attachment: isolation and characterization of mutants with impaired binding to vertebrate cells. J. Virol. 67, 3363-3374.

Dunn, E. F., and Connor, J. H. (2012). HijAkt: the PI3K/Akt pathway in virus replication and pathogenesis. Prog. Mol. Biol. Transl. Sci. 106, 223-250. doi: 10.1016/B978-0-12-396456-4.00002-X

Eliseeva, I. A., Kim, E. R., Guryanov, S. G., Ovchinnikov, L. P., and Lyabin, D. N. (2011). Y-box-binding protein 1 (YB-1) and its functions. Biochemistry 76, 1402-1433. doi: 10.1134/S0006297911130049

Foy, N. J., Akhrymuk, M., Akhrymuk, I., Atasheva, S., Bopda-Waffo, A., Frolov, I., et al. (2013). Hypervariable domains of nsP3 proteins of new world and old world alphaviruses mediate formation of distinct, virus-specific protein complexes. J. Virol. 87, 1997-2010. doi: 10.1128/JVI.02853-12

Frolov, I., Akhrymuk, M., Mobley, J. A., and Frolova, E. I. (2017). Hypervariable domain of eastern equine encephalitis virus nsP3 redundantly utilizes multiple cellular proteins for replication complex assembly. J. Virol. 91:e00371-17. doi: 10.1128/JVI.00371-17

Frolova, E., Gorchakov, R., Garmashova, N., Atasheva, S., Vergara, L. A., and Frolov, I. (2006). Formation of nsP3-specific protein complexes during Sindbis virus replication. J. Virol. 80, 4122-4134. doi: 10.1128/JVI.80.8.4122-4134.2006

Fros, J. J., Domeradzka, N. E., Baggen, J., Geertsema, C., Flipse, J., Vlak, J. M., et al. (2012). Chikungunya virus nsP3 blocks stress granule assembly by recruitment of G3BP into cytoplasmic foci. J. Virol. 86, 10873-10879. doi: 10.1128/JVI. 01506-12

Fros, J. J., Geertsema, C., Zouache, K., Baggen, J., Domeradzka, N., van Leeuwen, D. M., et al. (2015). Mosquito Rasputin interacts with chikungunya virus nsP3 and determines the infection rate in Aedes albopictus. Parasit. Vectors 8:464. doi: 10.1186/s13071-015-1070-4

Fros, J. J., and Pijlman, G. P. (2016). Alphavirus infection: host cell shut-off and inhibition of antiviral responses. Viruses 8:166. doi: 10.3390/v8060166

Froshauer, S., Karenbeck, J., and Helenius, A. (1988). Alphavirus RNA replicase is located on the cytoplasmic surface of endosomes and lysosomes. J. Cell Biol. 107, 2075-2086. doi: 10.1083/jcb.107.6.2075

Fullam, A., and Schröder, M. (2013). DExD/H-box RNA helicases as mediators of anti-viral innate immunity and essential host factors for viral replication. Biochim. Biophys. Acta 1829, 854-865. doi: 10.1016/j.bbagrm.2013.03.012

Galbraith, S. E., Sheahan, B. J., and Atkins, G. J. (2006). Deletions in the hypervariable domain of the nsP3 gene attenuate Semliki Forest virus virulence. J. Gen. Virol. 87, 937-947. doi: 10.1099/vir.0.81406-0

Garmashova, N., Gorchakov, R., Volkova, E., Paessler, S., Frolova, E., and Frolov, I. (2007). The old world and new world alphaviruses use different virus-specific proteins for induction of transcriptional shutoff. J. Virol. 81, 2472-2484. doi: 10.1128/JVI.02073-06

Goldfarb, S. B., Kashlan, O. B., Watkins, J. N., Suaud, L., Yan, W., Kleyman, T. R., et al. (2006). Differential effects of Hsc70 and Hsp70 on the intracellular trafficking and functional expression of epithelial sodium channels. Proc. Natl. Acad. Sci. U.S.A. 103, 5817-5822. doi: 10.1073/pnas.0507903103

Gomez de Cedrón, M., Ehsani, N., Mikkola, M. L., Garcı ìa, J. A., and Kääriäinen, L. (1999). RNA helicase activity of Semliki Forest virus replicase protein NSP2. FEBS Lett. 448, 19-22. doi: 10.1016/S0014-5793(99)00321-X

Gorchakov, R., Garmashova, N., Frolova, E., and Frolov, I. (2008). Different types of nsP3-containing protein complexes in Sindbis virus-infected cells. J. Virol. 82, 10088-10101. doi: 10.1128/JVI.01011-08

Gurer, C., Cimarelli, A., and Luban, J. (2002). Specific incorporation of heat shock protein 70 family members into primate lentiviral virions. J. Virol. 76, 4666-4670. doi: 10.1128/JVI.76.9.4666-4670.2002

Hahn, Y. S., Grakoui, A., Rice, C. M., Strauss, E. G., and Strauss, J. H. (1989). Mapping of RNA-temperature-sensitive mutants of Sindbis virus: complementation group F mutants have lesions in nsP4. J. Virol. 63, 1194-1202.
Hai, T., Yeung, M.-L., Wood, T. G., Wei, Y., Yamaoka, S., Gatalica, Z., et al. (2006). An alternative splice product of IкB kinase (IKK $\gamma$ ), IKK $\gamma-\Delta$, differentially mediates cytokine and human T-cell leukemia virus type 1 Tax-induced NF-кB activation. J. Virol. 80, 4227-4241. doi: 10.1128/JVI.80.9.4227-4241.2006

Hiscott, J., Nguyen, T. A., Arguello, M., Nakhaei, P., and Paz, S. (2006). Manipulation of the nuclear factor- $\kappa \mathrm{B}$ pathway and the innate immune response by viruses. Oncogene 2, 6844-6867. doi: 10.1038/sj.onc.1209941

Israël, A. (2010). The IKK complex, a central regulator of NF-кB activation. Cold Spring Harb. Perspect. Biol. 2:a000158. doi: 10.1101/cshperspect.a000158

Jee, H. (2016). Size dependent classification of heat shock proteins: a mini-review. J. Exerc. Rehabil. 12, 255-259. doi: 10.12965/jer.1632642.321

Jose, J., Snyder, J. E., and Kuhn, R. J. (2009). A structural and functional perspective of alphavirus replication and assembly. Future Microbiol. 4, 837-856. doi: 10. 2217/fmb.09.59

Jürchott, K., Bergmann, S., Stein, U., Walther, W., Janz, M., Manni, I., et al. (2003). YB-1 as a cell cycle-regulated transcription factor facilitating cyclin A and cyclin B1 gene expression. J. Biol. Chem. 278, 27988-27996. doi: 10.1074/jbc. M212966200

Karras, G. I., Kustatscher, G., Buhecha, H. R., Allen, M. D., Pugieux, C., Sait, F., et al. (2005). The macro domain is an ADP-ribose binding module. EMBO J. 24, 1911-1920. doi: 10.1038/sj.emboj.7600664

Kim, D. Y., Reynaud, J. M., Rasalouskaya, A., Akhrymuk, I., Mobley, J. A., Frolov, I., et al. (2016). New world and old world alphaviruses have evolved to exploit different components of stress granules, FXR and G3BP proteins, for assembly of viral replication complexes. PLOS Pathog. 12:e1005810. doi: 10.1371/journal. ppat. 1005810

Kloks, C. P., Spronk, C. A., Lasonder, E., Hoffmann, A., Vuister, G. W., Grzesiek, S., et al. (2002). The solution structure and DNA-binding properties of the coldshock domain of the human Y-box protein YB-1. J. Mol. Biol. 316, 317-326. doi: 10.1006/jmbi.2001.5334

LaStarza, M. W., Lemm, J. A., and Rice, C. M. (1994). Genetic analysis of the nsP3 region of Sindbis virus: evidence for roles in minus-strand and subgenomic RNA synthesis. J. Virol. 68, 5781-5791.

Lawrence, T. (2009). The nuclear factor NF-кB pathway in inflammation. Cold Spring Harb. Perspect. Biol. 1:a001651. doi: 10.1101/cshperspect. a001651

Li, L., Li, H. S., Pauza, C. D., Bukrinsky, M., and Zhao, R. Y. (2005). Roles of HIV1 auxiliary proteins in viral pathogenesis and host-pathogen interactions. Cell Res. 15, 923-934. doi: 10.1038/sj.cr.7290370

Liu, T., Daniels, C. K., and Cao, S. (2012). Comprehensive review on the HSC70 functions, interactions with related molecules and involvement in clinical diseases and therapeutic potential. Pharmacol. Ther. 136, 354-374. doi: 10.1016/ j.pharmthera.2012.08.014

Lobigs, M., Zhao, H. X., and Garoff, H. (1990). Function of Semliki Forest virus E3 peptide in virus assembly: replacement of E3 with an artificial signal peptide abolishes spike heterodimerization and surface expression of E1. J. Virol. 64, 4346-4355.

Lu, Z. H., Books, J. T., and Ley, T. J. (2005). YB-1 is important for late-stage embryonic development, optimal cellular stress responses, and the prevention of premature senescence. Mol. Cell. Biol. 25, 4625-4637. doi: 10.1128/MCB.25. 11.4625-4637.2005

Lusa, S., Garoff, H., and Liueström, P. (1991). Fate of the 6K membrane protein of Semliki Forest virus during virus assembly. Virology 185, 843-846. doi: 10.1016/ 0042-6822(91)90556-Q

Malet, H., Coutard, B., Jamal, S., Dutartre, H., Papageorgiou, N., Neuvonen, M., et al. (2009). The crystal structures of chikungunya and Venezuelan equine encephalitis virus nsP3 macro domains define a conserved adenosine binding pocket. J. Virol. 83, 6534-6545. doi: 10.1128/JVI.00189-09

Matsumoto, K., and Wolffe, A. P. (1998). Gene regulation by Y-box proteins: coupling control of transcription and translation. Trends Cell Biol. 8, 318-323. doi: 10.1016/S0962-8924(98)01300-2

McPherson, R. L., Abraham, R., Sreekumar, E., Ong, S.-E., Cheng, S.-J., Baxter, V. K., et al. (2017). ADP-ribosylhydrolase activity of Chikungunya virus macrodomain is critical for virus replication and virulence. Proc. Natl. Acad. Sci. U.S.A. 114, 1666-1671. doi: 10.1073/pnas.1621485114

Memmott, R. M., and Dennis, P. A. (2009). Akt-dependent and independent mechanisms of mTOR regulation in cancer. Cell. Signal. 21, 656-664. doi: 10.1016/j.cellsig.2009.01.004 
Mihailovich, M., Militti, C., Gabaldón, T., and Gebauer, F. (2010). Eukaryotic cold shock domain proteins: highly versatile regulators of gene expression. Bioessays 32, 109-118. doi: 10.1002/bies.200900122

Mohankumar, V., Dhanushkodi, N. R., and Raju, R. (2011). Sindbis virus replication, is insensitive to rapamycin and torin1, and suppresses Akt/mTOR pathway late during infection in HEK cells. Biochem. Biophys. Res. Commun. 406, 262-267. doi: 10.1016/j.bbrc.2011.02.030

Neuvonen, M., and Ahola, T. (2009). Differential activities of cellular and viral macro domain proteins in binding of ADP-ribose metabolites. J. Mol. Biol. 385, 212-225. doi: 10.1016/j.jmb.2008.10.045

Neuvonen, M., Kazlauskas, A., Martikainen, M., Hinkkanen, A., Ahola, T., and Saksela, K. (2011). SH3 domain-mediated recruitment of host cell amphiphysins by alphavirus nsP3 promotes viral RNA replication. PLOS Pathog. 7:e1002383. doi: 10.1371/journal.ppat.1002383

Oshiumi, H., Ikeda, M., Matsumoto, M., Watanabe, A., Takeuchi, O., Akira, S., et al. (2010). Hepatitis C virus core protein abrogates the DDX3 function that enhances IPS-1-mediated IFN-beta induction. PLOS ONE 5:e14258. doi: 10.1371 /journal.pone.0014258

Owonikoko, T. K., and Khuri, F. R. (2013). Targeting the PI3K/AKT/mTOR Pathway: Biomarkers of Success and Tribulation. Alexandria, VA: American Society of Clinical Oncology. doi: 10.1200/EdBook_AM.2013.33.e395

Panas, M. D., Ahola, T., and McInerney, G. M. (2014). The c-terminal repeat domains of nsP3 from the old world alphaviruses bind directly to G3BP. J. Virol. 88, 5888-5893. doi: 10.1128/JVI.00439-14

Panas, M. D., Schulte, T., Thaa, B., Sandalova, T., Kedersha, N., Achour, A., et al. (2015). Viral and cellular proteins containing FGDF motifs bind G3BP to block stress granule formation. PLOS Pathog. 11:e1004659. doi: 10.1371/journal.ppat. 1004659

Panas, M. D., Varjak, M., Lulla, A., Er Eng, K., Merits, A., Karlsson Hedestam, G. B., et al. (2012). Sequestration of G3BP coupled with efficient translation inhibits stress granules in Semliki Forest virus infection. Mol. Biol. Cell 23, 4701-4712. doi: 10.1091/mbc.E12-08-0619

Paranjape, S. M., and Harris, E. (2007). Y box-binding protein-1 binds to the dengue virus $3^{\prime}$-untranslated region and mediates antiviral effects. J. Biol. Chem. 282, 30497-30508. doi: 10.1074/jbc.M705755200

Park, E., and Griffin, D. E. (2009a). Interaction of Sindbis virus non-structural protein 3 with poly(ADP-ribose) polymerase 1 in neuronal cells. J. Gen. Virol. 90(Pt 9), 2073-2080. doi: 10.1099/vir.0.012682-0

Park, E., and Griffin, D. E. (2009b). The nsP3 macro domain is important for Sindbis virus replication in neurons and neurovirulence in mice. Virology 388, 305-314. doi: 10.1016/j.virol.2009.03.031

Patel, R. K., and Hardy, R. W. (2012). Role for the phosphatidylinositol 3-kinaseAkt-TOR pathway during Sindbis virus replication in arthropods. J. Virol. 86, 3595-3604. doi: 10.1128/JVI.06625-11

Peränen, J., and Kääriäinen, L. (1991). Biogenesis of type I cytopathic vacuoles in Semliki Forest virus-infected BHK cells. J. Virol. 65, 1623-1627.

Raj, G. V., Safak, M., MacDonald, G. H., and Khalili, K. (1996). Transcriptional regulation of human polyomavirus JC: evidence for a functional interaction between RelA (p65) and the Y-box-binding protein. YB-1. J. Virol. 70, 5944-5953.

Rathore, A. P., Haystead, T., Das, P. K., Merits, A., Ng, M. L., and Vasudevan, S. G. (2014). Chikungunya virus nsP3 \& nsP4 interacts with HSP-90 to promote virus replication: HSP-90 inhibitors reduce CHIKV infection and inflammation in vivo. Antiviral Res. 103, 7-16. doi: 10.1016/j.antiviral.2013.12.010

Rungrotmongkol, T., Nunthaboot, N., Malaisree, M., Kaiyawet, N., Yotmanee, P., Meeprasert, A., et al. (2010). Molecular insight into the specific binding of ADP-ribose to the nsP3 macro domains of chikungunya and Venezuelan equine encephalitis viruses: molecular dynamics simulations and free energy calculations. J. Mol. Graph. Model. 29, 347-353. doi: 10.1016/j.jmgm.2010. 09.010

Rupp, J. C., Sokoloski, K. J., Gebhart, N. N., and Hardy, R. W. (2015). Alphavirus RNA synthesis and non-structural protein functions. J. Gen. Virol. 96(Pt 9), 2483-2500. doi: 10.1099/jgv.0.000249

Santoro, M. G., Rossi, A., and Amici, C. (2003). NF-кB and virus infection: who controls whom. EMBO J. 22, 2552-2560. doi: 10.1093/emboj/ cdg267

Scholte, F. E. M., Tas, A., Albulescu, I. C., Žusinaite, E., Merits, A., Snijder, E. J., et al. (2015). Stress granule components G3BP1 and G3BP2 play a proviral role early in Chikungunya virus replication. J. Virol. 89, 4457-4469. doi: 10.1128/ JVI.03612-14

Schreiber, V., Dantzer, F., Ame, J. C., and De Murcia, G. (2006). Poly (ADPribose): novel functions for an old molecule. Nat. Rev. Mol. Cell Biol. 7, 517-528. doi: 10.1038/nrm1963

Schulte, T., Liu, L., Panas, M. D., Thaa, B., Dickson, N., Götte, B., et al. (2016). Combined structural, biochemical and cellular evidence demonstrates that both FGDF motifs in alphavirus nsP3 are required for efficient replication. Open Biol. 6:160078. doi: 10.1098/rsob.160078

Serva, S., and Nagy, P. D. (2006). Proteomics analysis of the Tombusvirus replicase: Hsp70 molecular chaperone is associated with the replicase and enhances viral RNA replication. J. Virol. 80, 2162-2169. doi: 10.1128/JVI.80.5.2162-21 69.2006

Shin, G., Yost, S. A., Miller, M. T., Elrod, E. J., Grakoui, A., and Marcotrigiano, J. (2012). Structural and functional insights into alphavirus polyprotein processing and pathogenesis. Proc. Natl. Acad. Sci. U.S.A. 109, 16534-16539. doi: 10.1073/pnas.1210418109

Spuul, P., Balistreri, G., Hellström, K., Golubtsov, A. V., Jokitalo, E., and Ahola, T. (2011). Assembly of alphavirus replication complexes from RNA and protein components in a novel trans-replication system in mammalian cells. J. Virol. 85, 4739-4751. doi: 10.1128/JVI.00085-11

Spuul, P., Balistreri, G., Kääriäinen, L., and Ahola, T. (2010). Phosphatidylinositol 3-kinase-, actin-, and microtubule-dependent transport of Semliki Forest virus replication complexes from the plasma membrane to modified lysosomes. J. Virol. 84, 7543-7557. doi: 10.1128/JVI.00477-10

Strauss, E. G., De Groot, R. J., Levinson, R., and Strauss, J. H. (1992). Identification of the active site residues in the nsP2 proteinase of Sindbis virus. Virology 191, 932-940. doi: 10.1016/0042-6822(92)90268-T

Strauss, J. H., and Strauss, E. G. (1994). The alphaviruses: gene expression, replication, and evolution. Microbiol. Rev. 58, 491-562.

Sunden, Y., Semba, S., Suzuki, T., Okada, Y., Orba, Y., Nagashima, K., et al. (2007). DDX1 promotes proliferation of the JC virus through transactivation of its promoter. Microbiol. Immunol. 51, 339-347. doi: 10.1111/j.1348-0421.2007. tb03907.x

Tamanini, F., Kirkpatrick, L. L., Schonkeren, J., van Unen, L., Bontekoe, C., Bakker, C., et al. (2000). The fragile X-related proteins FXR1P and FXR2P contain a functional nucleolar-targeting signal equivalent to the HIV-1 regulatory proteins. Hum. Mol. Genet. 9, 1487-1493. doi: 10.1093/hmg/9. 10.1487

Tamanini, F., Van Unen, L., Bakker, C., Sacchi, N., Galjaard, H., Oostra, B. A., et al. (1999). Oligomerization properties of fragile-X mental-retardation protein (FMRP) and the fragile-X-related proteins FXR1P and FXR2P. Biochem. J. 343(Pt 3), 517-523. doi: 10.1042/bj3430517

Thaa, B., Biasiotto, R., Eng, K., Neuvonen, M., Götte, B., Rheinemann, L., et al. (2015). Differential phosphatidylinositol-3-kinase-Akt-mTOR activation by Semliki Forest and Chikungunya viruses is dependent on nsP3 and connected to replication complex internalization. J. Virol. 89, 11420-11437. doi: 10.1128/ JVI.01579- 15

Ting, J. P., Painter, A., Zeleznik-Le, N. J., MacDonald, G., Moore, T. M., Brown, A., et al. (1994). YB-1 DNA-binding protein represses interferon gamma activation of class II major histocompatibility complex genes. J. Exp. Med. 179, 1605-1611. doi: 10.1084/jem.179.5.1605

Tossavainen, H., Aitio, O., Hellman, M., Saksela, K., and Permi, P. (2016). Structural basis of the high-affinity interaction between the alphavirus nonstructural protein-3 (nsP3) and the SH3 domain of amphiphysin-2. J. Biol. Chem. 291, 16307-16317. doi: 10.1074/jbc.M116.732412

Valiente-Echeverría, F., Hermoso, M. A., and Soto-Rifo, R. (2015). RNA helicase DDX3: at the crossroad of viral replication and antiviral immunity. Rev. Med. Virol. 25, 286-299. doi: 10.1002/rmv.184

Vara, J. Á. F., Casado, E., de Castro, J., Cejas, P., Belda-Iniesta, C., and GonzálezBarón, M. (2004). PI3K/Akt signalling pathway and cancer. Cancer Treat. Rev. 30, 193-204. doi: 10.1016/j.ctrv.2003.07.007

Varjak, M., Žusinaite, E., and Merits, A. (2010). Novel functions of the alphavirus nonstructural protein nsP3 c-terminal region. J. Virol. 84, 2352-2364. doi: 10. 1128/JVI.01540-09

Verma, S., Goyal, S., Jamal, S., Singh, A., and Grover, A. (2016). Hsp90: friends, clients and natural foes. Biochimie 127, 227-240. doi: 10.1016/j.biochi.2016. 05.018 
Vihinen, H., Ahola, T., Tuittila, M., Merits, A., and Kääriäinen, L. (2001). Elimination of phosphorylation sites of Semliki Forest virus replicase protein nsP3. J. Biol. Chem. 276, 5745-5752. doi: 10.1074/jbc.M006077200

Wang, H., and Ryu, W.-S. (2010). Hepatitis B virus polymerase blocks pattern recognition receptor signaling via interaction with DDX3: implications for immune evasion. PLOS Pathog. 6:e1000986. doi: 10.1371/journal.ppat. 1000986

Wang, R. Y.-L., Stork, J., and Nagy, P. D. (2009). A key role for heat shock protein 70 in the localization and insertion of Tombusvirus replication proteins to intracellular membranes. J. Virol. 83, 3276-3287. doi: 10.1128/JVI.02313-08

Wang, Y. F., Sawicki, S. G., and Sawicki, D. L. (1991). Sindbis virus nsP1 functions in negative-strand RNA synthesis. J. Virol. 65, 985-988.

Weng, Z., Rickles, R. J., Feng, S., Richard, S., Shaw, A. S., Schreiber, S. L., et al. (1995). Structure-function analysis of SH3 domains: SH3 binding specificity altered by single amino acid substitutions. Mol. Cell. Biol. 15, 5627-5634. doi: 10.1128/MCB.15.10.5627

Wigge, P., and McMahon, H. T. (1998). The amphiphysin family of proteins and their role in endocytosis at the synapse. Trends Neurosci. 21, 339-344. doi: 10.1016/S0166-2236(98)01264-8
Xu, L., Khadijah, S., Fang, S., Wang, L., Tay, F. P. L., and Liu, D. X. (2010). The cellular RNA helicase DDX1 interacts with Coronavirus nonstructural protein 14 and enhances viral replication. J. Virol. 84, 8571-8583. doi: 10.1128/JVI. 00392-10

Zárate, S., Cuadras, M. A., Espinosa, R., Romero, P., Juárez, K. O., CamachoNuez, M., et al. (2003). Interaction of rotaviruses with Hsc70 during cell entry is mediated by VP5. J. Virol. 77, 7254-7260. doi: 10.1128/JVI.77.13.7254-7260. 2003

Conflict of Interest Statement: The authors declare that the research was conducted in the absence of any commercial or financial relationships that could be construed as a potential conflict of interest.

Copyright (c) 2018 Lark, Keck and Narayanan. This is an open-access article distributed under the terms of the Creative Commons Attribution License (CC BY). The use, distribution or reproduction in other forums is permitted, provided the original author(s) or licensor are credited and that the original publication in this journal is cited, in accordance with accepted academic practice. No use, distribution or reproduction is permitted which does not comply with these terms. 